\section{Clonal hematopoiesis in patients with anti-neutrophil cytoplasmic antibody-associated vasculitis}

Clonal hematopoiesis of indeterminate potential (CHIP) - defined by the presence of a somatic hematologic cancer-associated gene mutation with a variant allele frequency of $\geq 2 \%$ - occurs in the peripheral blood of at least $10 \%$ of individuals older than 60 years of age without any history of a hematologic disorder. ${ }^{1,2}$ Mutations mainly affect epigenetic regulators of transcription $D N M T 3 A, T E T 2$, and ASXL1, leading to a competitive advantage of mutated hematopoietic stem cells with a subsequent differentiation bias toward the myeloid compartment. ${ }^{3,4}$ The frequency of CHIP increases with age and associates with higher risk of developing hematologic malignancies and cardiovascular diseases, leading to increased overall mortality.

Using a mouse model with Tet2-deficient macrophages, it was shown that atherosclerosis and coronary heart disease are driven by CHIP via an altered inflammasome function, leading to increased levels of pro-inflammatory cytokines. $^{6}$ Our group recently detected a correlation between DNMT3A mutations and chronic graft-versushost disease, providing further evidence of an important role of CHIP in chronic inflammatory reactions. ${ }^{7}$ However, little is known about the role of CHIP in autoimmune diseases. A study in 56 patients with rheumatoid arthritis showed no correlation of CHIP with disease activity. ${ }^{8}$ Anti-neutrophil cytoplasmic antibody (ANCA)-associated autoimmune vasculitides (AAV) comprise a variety of necrotizing vasculitides, including granulomatosis with polyangiitis and microscopic polyangi- itis, and are characterized by severe small vessel inflammation, potentially affecting every organ system. ANCA are directed against the autoantigens myeloperoxidase (MPO) and proteinase 3 (PR3). Upon binding to their cellsurface-expressed antigens, ANCA IgG provoke uncontrolled activation of neutrophils and monocytes, leading to endothelial damage and end-organ failure. In most individuals, the highest mutation burden of CHIP can be found in myeloid cells, ${ }^{4}$ which are the only autoantigenexpressing primary responder cells in AAV. Furthermore, TET2 and DNMT3A play a central role in gene silencing by regulating DNA methylation. In fact, defective gene silencing in myeloid cells from AAV patients has been reported. This dysregulated process included the ANCA autoantigens and correlated with relapse risk. ${ }^{9-11}$

In summary, recent data support the idea of potential links, regarding pathogenesis and clinical outcomes, between CHIP and autoimmune diseases/inflammatory conditions. We therefore characterized CHIP in a large cohort of patients with AAV, examining prevalence, dynamic changes over time, organ manifestations, ANCA antigen silencing, and ANCA-induced in vitro activation.

We collected peripheral blood samples from patients with AAV, seen at the Charité/HELIOS nephrology outpatient departments and wards (Berlin, Germany, between April 2005 and October 2018. The patients' demographic and clinical data were extracted from their medical records. All patients gave their written informed consent to inclusion in the study, which was conducted in accordance with the Declaration of Helsinki. Ethical approval was obtained from the local ethics committees.

Whole-blood DNA was screened for CHIP using a cus-
A

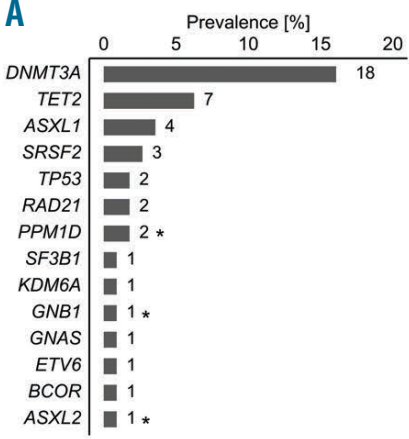

B

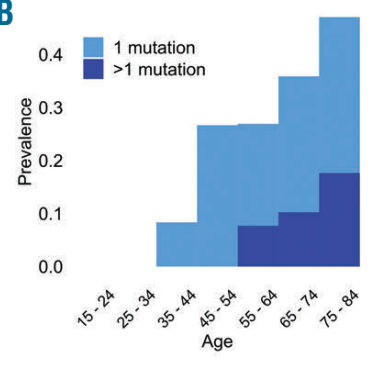

C

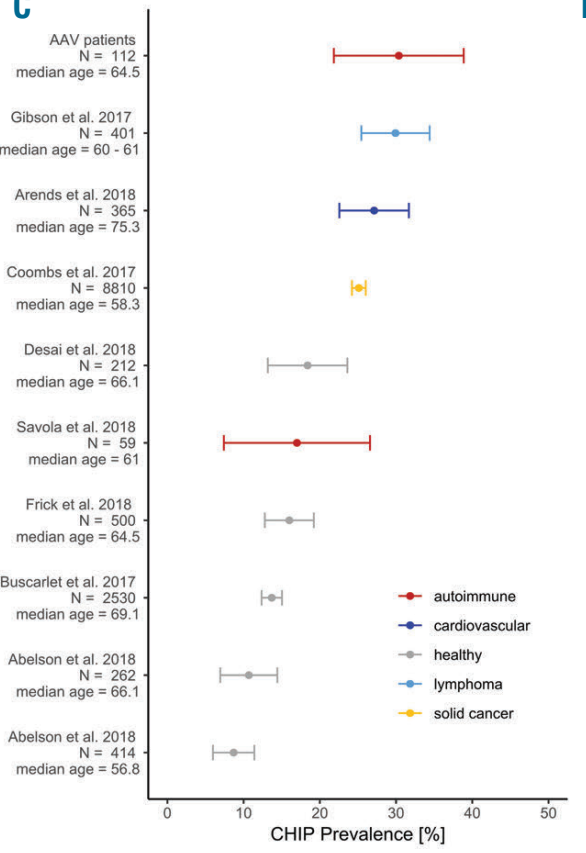

D

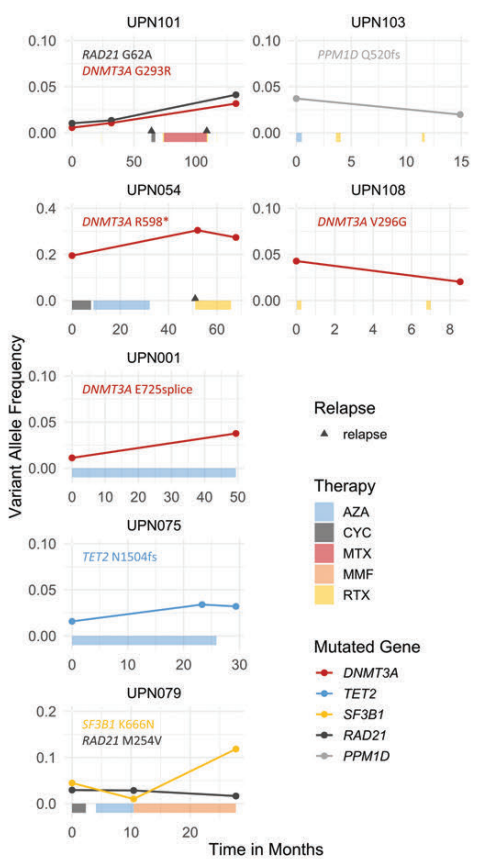

Figure 1. Sequencing analysis. (A) Spectrum of somatic gene mutations found in our cohort of 112 patients with ANCA-associated autoimmune vasculitis (AAV). Genes marked with an asterisk are only covered by the custom panel (Online Supplementary Methods). (B) Prevalence of clonal hematopoiesis of indeterminate potential (CHIP) according to age groups. Patients with a single mutation are represented in light blue, patients with multiple mutations in dark blue. (C) Comparison of CHIP prevalence in the AAV cohort with prevalences in previously described cohorts. Error bars depict the 95\% confidence intervals. (D) Longitudinal quantification of variant allele frequencies (VAF) in selected patients. Only patients with a relevant increase or decrease of VAF over time are shown. The therapy regimens administered are depicted as colored bars on the x-axis. Relapse is represented by triangles. UPN: unique patient number; AZA: azathioprine; CYC: cyclophosphamide; MTX: methotrexate; MMF: mycophenolate mofetil; RTX: rituximab. 
tomized version of the Illumina TruSight Myeloid Sequencing Panel (Online Supplementary Table S1) on a NextSeq sequencer. Sequencing analysis was performed using an Illumina BaseSpace platform sequencing HUB. Only nonsynonymous variants with allele frequencies $\geq 2 \%$ were included. Candidate variants were validated by targeted deep sequencing (Online Supplementary Methods). A total of 46 somatic mutations were identified in 34 out of 112 AAV patients $(30.4 \%)$ with a median variant allele frequency of $5.2 \%$ (Online Supplementary Table S2). While 25 patients had a single mutation, eight had two, and one patient had five. The most frequently mutated genes were DNMT3A (19/46=39,1\%), TET2 $(7 / 46=15.2 \%)$, and ASXL1 (4/46=8.7\%) (Figure 1A). Among the 46 mutations, 26 were missense, 18 were truncating and two were splice-site mutations. The most frequent base change in missense mutations was $\mathrm{C}>\mathrm{T}$ (16/30) (Online Supplementary Figure S1).

Compared to previously reported prevalences of CHIP in unselected control cohorts of similar age and sequencing technology, ${ }^{3,4,7,8,12-15}$ the CHIP prevalence in AAV patients was significantly higher $(30.4 \%$ vs. $13.5 \%$, $P<0.001)$ (Figure 1C, Online Supplementary Table S3). Bearing in mind the different sequencing technologies used in these studies, we investigated an age- and gender-matched control cohort of 112 healthy individuals, among whom 22 mutations were found in 20 subjects (healthy controls vs. AAV patients: $17.9 \%$ vs. $30.4 \%$, $P=0.042$ ) (Online Supplementary Table S4, Online
Supplementary Figures S2-S4). Of note, we found a relevant proportion of AAV patients with CHIP aged $\leq 55$ years $(6 / 33=18.2 \%)$ (Figure $1 \mathrm{~B})$. Follow-up peripheral blood samples were available for $19 \mathrm{CHIP}^{+} \mathrm{AAV}$ patients. The median follow-up was 2.3 years (range, 0.3-10.9 years). The mutational burden of serial samples from these 19 patients at two to four time-points was quantified by deep sequencing. ${ }^{4,7,16,17}$ While five patients showed a relevant increase in clone size, two patients had slightly decreasing clones and 12 patients showed no change in clone size over time (Figure 1D, Online Supplementary Figure S5). Next, we investigated one follow-up sample from each of $20 \mathrm{CHIP}^{-}$patients, collected 2 to 10 years after the initial sample. None of the 20 follow-up samples showed a new mutation.

Exploratory statistical analyses were performed to identify associations between CHIP and clinical parameters (76 patients with granulomatosis with polyangiitis and 34 with microscopic polyangiitis). $\mathrm{CHIP}^{+}$patients were significantly older than $\mathrm{CHIP}^{-}$patients (median 70.5 vs. 63.0 years, respectively, $P=0.017$ ). The prevalence of CHIP was not higher among patients who had received immunosuppressive treatment prior to sampling (100\% steroids, $90 \%$ cyclophosphamide, $20 \%$ rituximab, 16\% azathioprine, $13 \%$ methotrexate). No differences in blood counts, red cell distribution width, creatinine levels, comorbidities, the development of malignancies, disease activity status, and AAV relapse risk were observed with respect to CHIP status. However, disease manifesta-
A

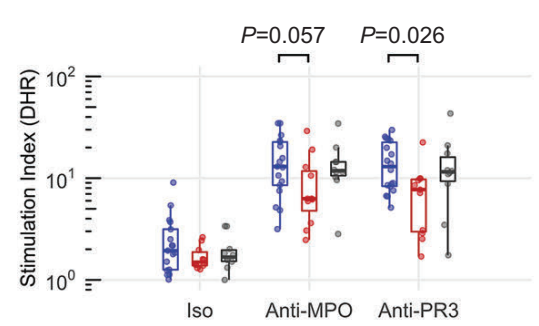

B

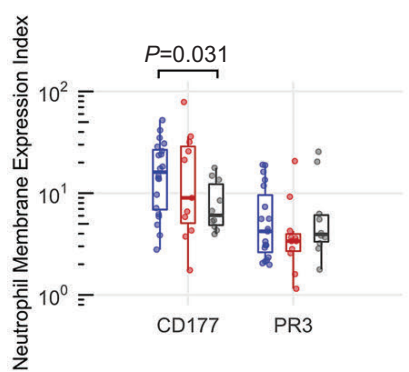

C

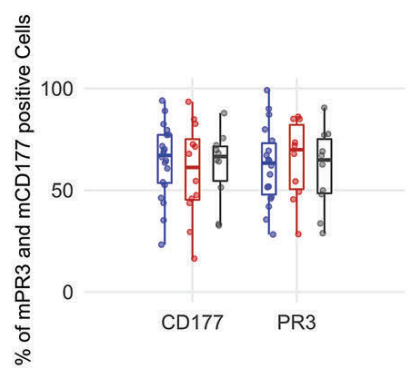

D

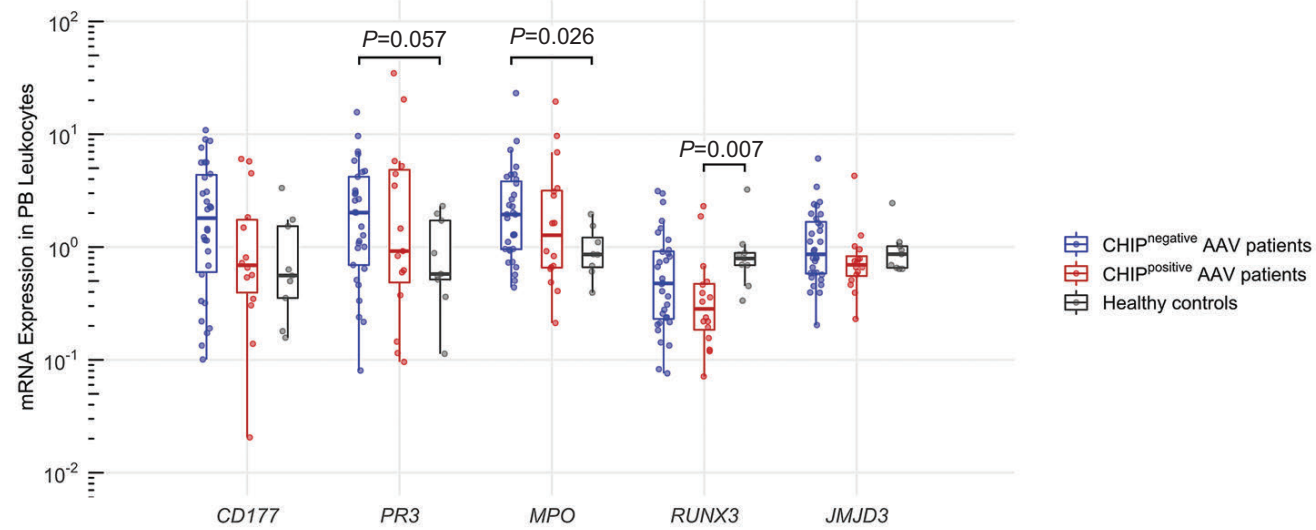

Figure 2. Functional data. Individual data points are depicted in scatterplots and summarized in boxplots. (A) Neutrophil oxidative burst detection using the DHR assay; depicted is the stimulation index $\mathrm{SI}=$ mean channel fluorescence of stimulated versus unstimulated cells, (B, C) Neutrophil membrane expression of CD177 or PR3 measured on isolated neutrophils by flow cytometry using anti-NB1 or anti-PR3 antibodies, depicted as expression index El (B) or percentage of mPR3- and CD177-positive cells (C). El = (MFlstimulated cells- MFlunstimulated cells)/MFlunstimulated cells. (D) mRNA expression measured in PB leukocytes with qPCR. (m)PR3: (membrane-)proteinase 3; MPO: myeloperoxidase; RUNX3: Runt-related transcription factor 3; JMJD3: jumonji domain-containing protein 3; DHR: dihydrorhodamine; NB1: neutrophil-specific antigen; PB: peripheral blood; El: expression index; SI: stimulation index; MFI: mean fluorescence intensity. 
tion patterns differed: $\mathrm{CHIP}^{+}$patients affected by granulomatosis with polyangiitis showed less renal disease (68.2\% vs. 88.5\%, $P=0.049)$ and nervous system involvement ( $0 \%$ vs. $19.2 \%, P=0.028$ ) (Online Supplementary Tables S5-S8, Online Supplementary Figures S6-S8).

Next, we aimed to investigate ANCA antigen silencing and ANCA-induced in vitro activation. To this aim, in vitro neutrophil stimulation assays using dihydrorhodamine oxidation with monoclonal antibodies against the ANCA antigens $\mathrm{MPO}$ and PR3 were performed in a subset of AAV patients and healthy controls (Online Supplementary Methods) who had tested negative for CHIP. A reduced activation was observed in $\mathrm{CHIP}^{+}$compared to $\mathrm{CHIP}^{-}$ AAV patients (anti-MPO: stimulation index: 6.29 vs. 13.01, $P=0.057$; anti-PR3: stimulation index $7.72 \mathrm{vs.}$ 13.00, $P=0.026$ ) (Figure 2A), whereas no difference in membrane expression index or percentage of positive cells was observed (Figure 2B, C). In addition, peripheral blood mRNA levels of $P R 3, M P O, C D 177, R U N X 3$, and $J M J D 3$ were measured by quantitative polymerase chain reaction. $\mathrm{CHIP}^{-} \mathrm{AAV}$ patients showed increased expression of $M P O$ and $P R 3$ mRNA compared to levels in healthy controls (MPO: 1.94 vs. $0.86, P=0.026$; PR3: 2.02 vs. $0.58, P=0.057$ ), a difference that was less apparent in $\mathrm{CHIP}^{+}$patients. However, $\mathrm{CHIP}^{+} \mathrm{AAV}$ patients showed reduced expression of $R U N X 3 \mathrm{mRNA}$ compared to the level in healthy controls (0.28 vs. $0.79, P=0.007)$ (Figure 2). Due to small numbers of patients, we were not able to further subdivide $\mathrm{CHIP}^{+} \mathrm{AAV}$ patients according to affected genes or variant allele frequencies and could not therefore evaluate their potential impact on our findings (Online Supplementary Table S9). Additionally, significant differences in neutrophil and lymphocyte counts between AAV patients and healthy controls might have affected our results and limit the ability to draw generalized conclusions (Online Supplementary Table S10).

In summary, we detected CHIP in 34 out of 112 patients $(30.4 \%)$, a significantly higher prevalence than reported in healthy cohorts and in our age-matched control group, but comparable to increased frequencies reported in patients with cancer, ${ }^{12}$ aplastic anemia ${ }^{18}$ and cardiovascular disease. ${ }^{5}$ While altered inflammatory signaling has been proposed as a mechanism underlying the association of myelodysplastic syndromes with autoimmune diseases/inflammatory conditions, ${ }^{19}$ a similar mechanism might link CHIP with such conditions and, in particular, with AAV. Dysregulated ANCA autoantigen transcription is commonly observed in AAV and could be altered by $\mathrm{CHIP}$. Interestingly, $\mathrm{CHIP}^{-}$, but not $\mathrm{CHIP}^{+} \mathrm{AAV}$ patients showed upregulation of autoantigen mRNA expression that was previously reported..$^{9-11}$ This rather surprising finding suggests that the upregulated ANCA antigen expression is presumably a secondary phenomenon in AAV, induced by inflammatory signaling which is defective in $\mathrm{CHIP}^{+}$cells. In line with this, reduced ANCAinduced neutrophil activation was observed in $\mathrm{CHIP}^{+}$ patients. Interestingly, we have previously demonstrated that ANCA-induced production of reactive oxygen species plays a major role in downregulating inflammasome activation by oxidative inhibition of the inflammasome-caspase-1-interleukin- $1 \beta$ cascade..$^{20}$ The diminished production of reactive oxygen species by $\mathrm{CHIP}^{+}$neutrophils that we found could, therefore, contribute to an overacting activation of the inflammasome and thereby affect the pathogenesis of AAV. Clinically, we found fewer renal and neuronal manifestations in $\mathrm{CHIP}^{+}$ patients, supporting the idea that CHIP functions as a disease modifier in AAV.
In longitudinal analysis, more than $25 \%$ of patients showed an increase in clone size over time without any significant impact of a specific treatment on clone expansion. CHIP frequency was not increased in patients previously treated with immunosuppressive/cytotoxic agents and not enriched for mutations involved in DNA damage response (Online Supplementary Table S11). It therefore appears unlikely that the high prevalence of CHIP is merely a consequence of cytotoxic treatment and, together with the expanding clone sizes, warrants a closer monitoring of affected AAV patients because of the known risk of progression to myelodysplastic syndromes or acute myeloid leukemia. ${ }^{13,15}$

Collectively, our data reveal a new association of AAV with CHIP with potentially disease-modifying effects as shown for neutrophil activation, autoantigen transcription regulation and organ manifestation. We acknowledge that, given the multiple tests, the $P$-values do not picture the global type I error. Future studies and functional investigations are now warranted to confirm these results and decipher the molecular mechanisms.

Christopher Maximilian Arends, ${ }^{1 *}$ Marlene Weiss, ${ }^{2,3^{*}}$

Friederike Christen, ${ }^{1}$ Claudia Eulenberg-Gustavus, ${ }^{2}$

Anthony Rousselle, ${ }^{2}$ Ralph Kettritz, ${ }^{2,3}$ Kai-Uwe Eckardt, ${ }^{3}$

Willy Chan, ${ }^{1}$ Kaja Hoyer, ${ }^{1}$ Mareike Frick, ${ }^{1}$ Lars Bullinger, ${ }^{1,4}$ Markus Bieringer, 5 Arian Schreiber ${ }^{2,3 \#}$ and Frederik Damm ${ }^{1,4 \#}$

**AMA and $M W$ contributed equally as co-first authors.

${ }^{*} A S$ and FD contributed equally as co-senior authors.

Charité - Universitätsmedizin Berlin, corporate member of Freie Universität Berlin, Humboldt-Universität zu Berlin, and Berlin Institute of Health, Department of Hematology, Oncology, and Tumor Immunology, Berlin; 'Experimental and Clinical Research Center, Charité, Max Delbrück Center for Molecular Medicine in the Helmholtz Association, Berlin; ${ }^{3}$ Charité - Universitätsmedizin Berlin, Department of Nephrology and Intensive Care Medicine, Berlin; ${ }^{4}$ German Cancer Consortium (DKTK) and German Cancer Research Center (DKFZ), Heidelberg and 'HELIOS Klinikum Berlin-Buch, Department of Cardiology and Nephrology, Berlin, Germany

Correspondence: FREDERIK DAMM - frederik.damm@charite.de ADRIAN SCHREIBER - adrian.schreiber@charite.de

doi:10.3324/haematol.2019.223305

Funding: this study was supported by a grant from the Dr. Werner Jackstädt Foundation awarded to AS and FD, grant \#DA1787/1-1 from the Deutsche Forschungsgemeinschaft, a Mechthild-Harf research fellowship from the DKMS Giving Life Foundation, and grant \#2017_EKES.33 from the Else Kröner-Fresenius-Stiftung awarded to FD, grant SCHR 771/8-1 from the Deutsche Forschungsgemeinschaft to $A S$, and grant 394046635 - SFB 1365 from the Deutsche Forschungsgemeinschaft to $A S$ and $R K$; ECRC grants to AS and RK; $C M A$ received a fellowship from the Berlin Institute of Health, and WC received a fellowship from the Deutsche José Carreras LeukämieStiftung.

Information on authorship, contributions, and financial \& other disclosures was provided by the authors and is available with the online version of this article at WWw. haematologica.org.

\section{References}

1. Genovese G, Kahler AK, Handsaker RE, et al. Clonal hematopoiesis and blood-cancer risk inferred from blood DNA sequence. N Engl J Med. 2014;371(26):2477-2487.

2. Steensma DP, Bejar R, Jaiswal S, et al. Clonal hematopoiesis of indeterminate potential and its distinction from myelodysplastic syndromes. Blood. 2015;126(1):9-16.

3. Buscarlet M, Provost S, Zada YF, et al. DNMT3A and TET2 dominate clonal hematopoiesis and demonstrate benign phenotypes and different genetic predispositions. Blood. 2017;130(6):753-762 
4. Arends CM, Galan-Sousa J, Hoyer K, et al. Hematopoietic lineage distribution and evolutionary dynamics of clonal hematopoiesis. Leukemia. 2018;32(9):1908-1919.

5. Jaiswal S, Natarajan P, Silver AJ, et al. Clonal hematopoiesis and risk of atherosclerotic cardiovascular disease. $N$ Engl J Med. 2017;377(2):111-121.

6. Fuster JJ, MacLauchlan S, Zuriaga MA, et al. Clonal hematopoiesis associated with TET2 deficiency accelerates atherosclerosis development in mice. Science. 2017;355(6327):842-847.

7. Frick M, Chan W, Arends CM, et al. Role of donor clonal hematopoiesis in allogeneic hematopoietic stem-cell transplantation. J Clin Oncol. 2019;37(5):375-385.

8. Savola P, Lundgren S, Keranen MAI, et al. Clonal hematopoiesis in patients with rheumatoid arthritis. Blood Cancer J. 2018;8(8):69.

9. Ciavatta DJ, Yang J, Preston GA, et al. Epigenetic basis for aberrant upregulation of autoantigen genes in humans with ANCA vasculitis. J Clin Invest. 2010;120(9):3209-3219.

10. Jones BE, Yang J, Muthigi A, et al. Gene-specific DNA methylation changes predict remission in patients with ANCA-associated vasculitis. J Am Soc Nephrol. 2017;28(4):1175-1187.

11. McInnis EA, Badhwar AK, Muthigi A, et al. Dysregulation of autoantigen genes in ANCA-associated vasculitis involves alternative transcripts and new protein synthesis. J Am Soc Nephrol. 2015;26(2):390-399.

12. Coombs CC, Zehir A, Devlin SM, et al. Therapy-related clonal hematopoiesis in patients with non-hematologic cancers is common and associated with adverse clinical outcomes. Cell Stem Cell. 2017;21(3):374-382.e4.

13. Desai P, Mencia-Trinchant N, Savenkov O, et al. Somatic mutations precede acute myeloid leukemia years before diagnosis. Nat Med. 2018;24(7):1015-1023.

14. Gibson CJ, Lindsley RC, Tchekmedyian V, et al. Clonal hematopoiesis associated with adverse outcomes after autologous stem-cell transplantation for lymphoma. J Clin Oncol. 2017;35(14):1598-1605

15. Abelson S, Collord G, Ng SWK, et al. Prediction of acute myeloid leukaemia risk in healthy individuals. Nature. 2018;559(7714):400404.

16. Christen F, Hoyer K, Yoshida K, et al. Genomic landscape and clonal evolution of acute myeloid leukemia with $t(8 ; 21)$ : an international study on 331 patients. Blood. 2019;133(10):1140-1151.

17. Damm F, Mylonas E, Cosson A, et al. Acquired Initiating mutations in early hematopoietic cells of CLL patients. Cancer Discov. 2014;4(9):1088-1101

18. Yoshizato T, Dumitriu B, Hosokawa K, et al. Somatic mutations and clonal hematopoiesis in aplastic anemia. $N$ Engl J Med. 2015;373(1):35-47.

19. Sallman DA, List $\mathrm{A}$. The central role of inflammatory signaling in the pathogenesis of myelodysplastic syndromes. Blood. 2019;133(10):1039-1048.

20. Schreiber A, Luft FC, Kettritz R. Phagocyte NADPH oxidase restrains the inflammasome in ANCA-induced GN. J Am Soc Nephrol. 2015;26(2):411-424. 